\title{
A Robust Technique without Additional Computational Cost in Evolutionary Antenna Optimization
}

\author{
Caie $\mathrm{Hu}$, Sanyou Zeng, Yuhong Jiang, Jianqing Sun, Yongzhi Sun, and Steven Gao ( Member, IEEE),
}

\begin{abstract}
A robustness-enhancing technique without additional computational cost in antenna optimization design is presented. The robustness is implemented by minimizing the variances of the gains, axial ratios and VSWRs over the required frequency band. It is demonstrated that the new technique has two obvious advantages. One is that it can ensure the antenna robustness without the extra computational overhead. The other one is that it is possible to broaden the bandwidth of the antenna. We apply this technique to design a microstrip antenna at $2.4 \mathrm{GHz}$. Experimental results show that, by adopting this new technique, the evolved antenna is more robust than by using two other techniques.
\end{abstract}

Keywords: evolutionary algorithms, antenna design, robust optimization, constrained optimization.

\section{INTRODUCTION}

A NTENNA design problems are usually modeled as constrained optimization problems (COPs) [1, 2], which is usually solved by evolutionary algorithms (EAs), including Genetic Algorithms (GAs) [3], Differential Evolutions (DEs) [4], Evolution Strategies (ESs) [5], Particle Swarm Optimizations (PSOs) [6] and other evolutionary techniques.

EAs are widely applied to optimize geometric shape of antennas, mainly including the shape of an antenna and the space between antenna elements of an array. It is worth noting that most evolutionary antenna researches are mainly involved in optimizing the space between antenna elements of an array while optimizing the shape of an antenna is relatively few. This is mainly because optimizing the shape of an antenna is very expensive due to the time-consuming electromagnetic simulation while optimizing the space between antenna elements of an array is cheap for the analytical array factor. This paper focuses on only the optimization of the shape of an antenna.

In optimizing the shape of an antenna, EAs begin with a randomly initialized parent population of antennas with arbitrary shapes within the first generation. In each iteration during

Manuscript received September 19, 2017; revised September 28, 2017, June 20, 2018, October 16, 2018. This work was supported by the National Natural Science Foundation of China and other foundations (No.s: 61673355, 61271140, 61706102), Natural Science Foundation of Shanxi Province(No.s: ZR2017MF042, ZR2017QF012), and the high-performance computing platform of China University of Geosciences.

Caie Hu (1943327486@qq.com), Sanyou Zeng (sanyouzeng@gmail.com, Corresponding author) and Yuhong Jiang (yuhongjiang1026@gmail.com) are with the School of Mechanical Engineering and Electronic Information, China University of Geoscience,430074 Wuhan, China.

Jianqing Sun (sunjianqing126@126.com), Yongzhi Sun (nanshen01@126.com, Corresponding author) are with No. 8511 Research Institute of CASIC, 210007 Nanjing, China.

S. Gao (s.gao@kent.ac.uk) is with the Department of Engineering and Digital Arts, University of Kent, CT2 7NZ Canterbury, UK. middle generations, an offspring population is generated via a number of variation operators and each individual in it is evaluated by electromagnetic simulate software, after which the new parent population for the next iteration is selected from the offspring population or a combination of the parent and offspring population. In the last generation, the optimization result is selected from the current population. The general process of evolutionary antenna optimization is shown in Fig. 1. There are some literatures work on this topic. GAs are often used to optimize the shape of an antenna. Patch antennas in [7] obtained a pentaband design covering GSM1800, GSM1900, $U M T S, L T E 2300$ and Bluetooth bands with fractional bands about $38 \%$. Wire antenna in [8] obtained a good result that quality factors $(Q s)$ obtained has increased from 15.8 to 590. Also, the evolved wire antennas achieved a high gain across a wider range of elevation angles in [9], etc; Similarly, ESs also perform well on the issue. A patch antenna has been successfully optimized in [10] which achieved a good impedance matching and radiation characteristics in the entire band of $W L A N$ (IEEE 802.11 protocol). The performances of patch antenna in [11] have been improved, etc; Also, PSOs do well in this aspect. The bandwidth of patch antenna in [12] was broadened by $54 \%$. The bandwidth and gain of planar antenna in [13] have been promoted, etc; Moreover, DEs are no exception. Patch antenna in [14] achieved an effective bandwidth of $9 \%$. Wire antenna in [15] achieved a good impedance matching. The curve fitting based DE and the cuckoo search optimization focused on enhancing bandwidth of microstrip patch antenna in [16]. Compact MIMO antennas provided isolation higher than $30 \mathrm{~dB}$ in a relative bandwidth of $40 \%$ in [17], etc.

Robust design is very important in engineering fields. It is a hot topic in evolutionary computation. Some previously published methods on robust evolutionary optimization have been presented in [18-25]. In [18], the method of adopting the average fitness value of an individual's neighbors instead of the fitness value of that individual. It applied normal distribution to generate neighbors. Thus the cost of assessing each individual increases several times. Another robust method was proposed by Zeng in [19], which adopted orthogonal array to generate neighbors and also used the average fitness value of the neighbors as the individual's objective value. The obvious advantage is the number of neighbors is less than in [18]. Both above methods need neighbors for the individual's assessment, which increases time cost. Besides that, Paenke in [20] suggested to construct computationally 


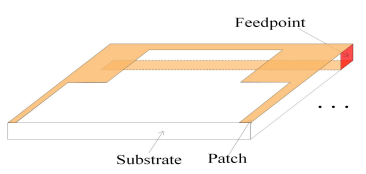

First generation

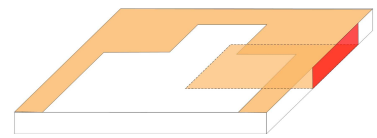

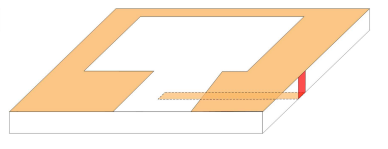

Middle generations
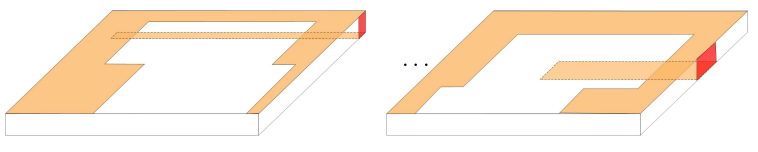

Last generation

Fig. 1. Sequence of process for evolutionary antenna optimization:EAs begin with arbitrary antennas in the first generation, iterate during middle generations, select the optimized antenna in the last generation.

efficient models associated with available solutions to replace the expensive fitness function in calculating the mean and variance of neighbors. However, the process of modeling still took extra time. In paper [21], an active robust optimization was presented as a new robust optimization approach. It considered products that are able to adapt to environmental changes. But, for a proper evaluation of an adaptive solution, it has to be assessed for each scenario with its best possible performance. Authors proposed to find robust solutions for DOPs to account for a solution's future fitness explicitly in [22], which were formulated as the robust optimization over time (ROOT) problem. In order to find optimal robust solutions in terms of average fitness or survival time, a solution's future fitness needs to be predicted, it is important to build a learning model for predictive tasks, but it needs additional computational cost. In paper [23], the random solutions were generated using the Latin Hyper Sphere (LHS), it also did not solve the problem of reducing additional computational cost. Authors proposed robust optimization, which considered an efficient means to identify the set of tradeoff robust solutions with an affordable computational cost in [24]. However, reduced computational cost is a less important factor to consider. A generic multi-objective optimization framework for robust optimization over time that simultaneously maximizes the robustness and minimizes the switching cost was proposed in [25]. The predicted fitness of the current solutions in a future environment according to their fitness values in the current and previous, based on which the predictor can be constructed, however, predictor construction also need additional time in the paper. Notably, we did not find any evolutionary robust optimization of antennas.

The above robustness techniques require additional computational overhead. The reason why evolutionary optimization of antennas is usually very expensive is that the antenna simulation is very expensive. The evolutionary robust optimization of antenna shapes is even more expensive with the additional computational overhead. That is why we could find no evolutionary robust optimization of antennas. In this paper, we propose a technique to enhance robustness technique for evolutionary antenna design without extra computational overhead. The electromagnetic characteristics (the shape of the directive pattern, the front-to-rear ratio, the input impedance, and so on) vary significantly with frequency. A higher frequency antenna usually requires a smaller size of the geometrical structure. To some extent, the smaller variances of electromagnetic characteristics over frequency band mean that the electromagnetic characteristics have a weaker dependence on the frequency and the geometrical structure. An antenna with such a weak dependence can have a broad frequency band and be insensitive to the geometrical structure, which means this antenna is robust. Minimizing these variances can achieve a weakest dependence. So the antenna with minimum variances can be the one with a broad frequency band and robustness. To obtain such an antenna, the sum of these variances is chosen as the objective function of the constrained optimization problem modeled for antenna design in this paper.

The rest of this paper is structured as follows. Section II introduces some related work. Section III proposes our robust technique. We compare the performance of the proposed objective technique with other two objective techniques in section IV. Finally, Section V summarizes the conclusion of this paper.

\section{RELATED WORK}

An antenna problem is usually modeled as a constrained optimization problem (COP), which is classified as a nonlinear problem. Traditional optimizers could not solve the problem well while evolutionary algorithms have potentiality in solving such complex problem. Differential evolution (DE) is employed in this paper.

We provide a review over the concept of the COP and also the differential evolution (DE).

In this paper, we suppose the COP as Minimize Optimization.

\section{Definition 1: (Constrained Optimization Problem (COP))}

A general COP includes an objective function, a set of $\mathrm{m}$ constraints and a set of $n$ variables. The objective function and constraints are functions of the variables. A COP can be mathematically defined as:

$$
\begin{array}{ll}
\min & y=f(\vec{x}) \\
\text { st: } & \vec{g}(\vec{x})=\left(g_{1}(\vec{x}), g_{2}(\vec{x}), \ldots, g_{m}(\vec{x})\right) \leq \overrightarrow{0} \\
\text { where } & \vec{x}=\left(x_{1}, x_{2}, \ldots, x_{n}\right) \in \mathbf{X} \\
& \mathbf{X}=\{\vec{x} \mid \vec{l} \leq \vec{x} \leq \vec{u}\} \\
& \vec{l}=\left(l_{1}, l_{2}, \ldots, l_{n}\right), \vec{u}=\left(u_{1}, u_{2}, \ldots, u_{n}\right)
\end{array}
$$

where $f(\vec{x})$ is the objective function, $\vec{g}(\vec{x}) \leq \overrightarrow{0}$ is the constraint, $\overrightarrow{0}$ is the constrained boundary. $\vec{x}$ is the solution vector and $\mathbf{X}$ denotes the solution space, $\vec{l}$ and $\vec{u}$ are the lower bound and upper bound of the solution space.

Definition 2: (Feasible solution and Feasible set )

A solution $\vec{x}=\left(x_{1}, x_{2}, \ldots, x_{n}\right) \in \mathbf{X}$ is said feasible, if $\vec{g}(\vec{x}) \leq \overrightarrow{0}$. The Feasible set of a COP is defined as:

$$
S_{F}=\{\vec{x}: \vec{x} \in \mathbf{X}, \vec{g}(\vec{x}) \leq \overrightarrow{0}\}
$$

\section{Definition 3: (Constrained Violation )}


Given a solution $\vec{x}$, the Constrained Violation of a constraint in Eq. (1) is usually defined as:

$$
G_{i}(\vec{x})=\max \left\{g_{i}(\vec{x}), 0\right\}, i=1,2, \ldots, m .
$$

\section{Definition 4: (Solution Violation )}

Given a solution $\vec{x}$, the Solution Violation $\psi(\vec{x})$ is defined as:

$$
\psi(\vec{x})=\frac{1}{m} \sum_{i=1}^{m} \frac{G_{i}(\vec{x})}{\max _{\vec{x} \in P(0)}\left\{G_{i}(\vec{x})\right\}}
$$

Where $\mathrm{P}(0)$ is the initial population of an EA, if $\max _{\vec{x} \in P(0)}\left\{G_{i}(\vec{x})\right\}<1, i=1,2, \ldots, m$, we replace the $\max _{\vec{x} \in P(0)}\left\{G_{i}(\vec{x})\right\}$ with 1 .

The order of two solutions is usually given as the Algorithm 1.

Algorithm 1 Comparison of $\vec{x}_{1}$ and $\vec{x}_{2}$
Case1 Both are feasible, then the one with smaller objective
f wins
Case 2 One is feasible and the other is infeasible, then the
feasible one wins
Case3 Both are infeasible, then the one with smaller viola-
tion $\Psi$ wins.

The differential evolution (DE) has many different schemes. The basic DE strategy DE/rand/1/bin is adopted in this paper. The performance of the differential evolution and its other versions were tested by 24 benchmark problems [26]. The results were competitive to state-of-the-art algorithms in solving continuous constrained problems. In this paper, differential evolution with the strategy DE/rand/1/bin is employed to the problem.

The comparison operator is to determine which one is better in comparison of two solutions which is shown as Algorithm 1 .

\section{PRoposed Robust TECHNiQUE}

We present a microstrip planar dipole antenna to illustrate our technique. The microstrip antenna design is modeled as a COP. Our robust technique is presented in constructing the objective function in the COP.

\section{A. Design requirements of the microstrip patch antenna}

The requirements of microstrip planar dipole antenna are shown in Table I:

TABLE I

DESIGN REQUIREMENTS OF MICROSTRIP PLANAR DIPOLE ANTENNA

\begin{tabular}{|c|c|}
\hline Frequency & $2400 \pm 50 \mathrm{MHz}$ \\
\hline Input Impedance & $50 \Omega$ \\
\hline VSWR & $\leq 2$ \\
\hline Polarization Mode & linear polarization \\
\hline \multirow{2}{*}{ Gain } & $\geq 0 d B$ \\
\cline { 2 - 2 } & $-180^{\circ} \leq \theta \leq 180^{\circ}, \varphi=90^{\circ}$ \\
\hline
\end{tabular}

In general, the antenna design when the smaller VSWR accompanied with higher gain is the better design.

\section{B. Parametric antenna structure}

The parametric structure of the microstrip planar dipole antenna is shown in Fig. 2:

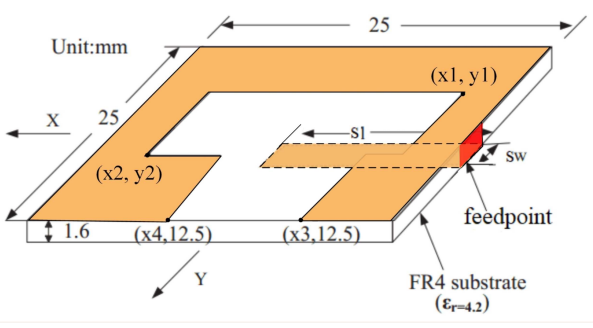

Fig. 2. Parametric structure of microstrip patch antenna

As shown in Fig. 2, the planar dipole antenna contains three parts: the substrate, the metal patch printed on the top side of the substrate, and the metal microstrip line printed on the bottom side of the substrate.

The substrate is a cuboid with a size of $25 \mathrm{~mm} \times 25 \mathrm{~mm} \times$ $1.6 \mathrm{~mm}$ and the relative permittivity is 4.2 . Starting from the front edge of the top metal patch, two adjacent rectangles are cut in the patch. The metal microstrip line is a narrow long rectangle. The feed point is at the center of the right edge of the antenna. Both the location and the size of the patch and the microstrip line are parameterized.

\section{Solution vector and solution space}

The shape of the top patch is determined by four points $\left(x_{1}, y_{1}\right),\left(x_{2}, y_{2}\right),\left(x_{3}, 12.5\right)$ and $\left(x_{4}, 12.5\right)$, and the shape of the bottom microstrip line is determined by $s l$ and $s w$ as shown in Fig. 2.

All these eight variables $\vec{x}=\left(s l, s w, x_{1}, y_{1}, x_{2}, y_{2}, x_{3}, x_{4}\right)$ make up of the solution vector, and the solution space is the ranges of the eight variables, see Eq. (5).

$$
\begin{aligned}
& \mathbf{X}=\{\vec{x} \mid \vec{l} \leq \vec{x} \leq \vec{u}\} \\
& \vec{x}=\left(s l, s w, x_{1}, y_{1}, x_{2}, y_{2}, x_{3}, x_{4}\right) \\
& \vec{l}=(2,2,-11.5,-11.5,0,0,-11.5,0) \\
& \vec{u}=(25,6,0,0,11.5,11.5,0,11.5)
\end{aligned}
$$

Ranges of these structure variables, i.e., the solution space, are shown in Table II.

\section{Objective and Constraints}

1) Objective and Details of Our technique: In this paper, our technique is to construct an objective function to enhance the robustness of the antenna. The objective $f(\vec{x})$ is defined as the sum of variances over frequency band, see Eq. (6):

$$
\begin{aligned}
& f(\vec{x})=\sum_{\theta} \sum_{\varphi}\left(\text { Gvariance }_{(\theta, \varphi)}\right. \\
& +
\end{aligned}
$$

where $(\theta, \varphi)$ represents one direction in spherical coordinates. $\theta$ is the elevation and $\varphi$ is the azimuth. Ranges of $\theta$ 
TABLE II

RANGES OF STRUCTURE VARIABLES

\begin{tabular}{|c|c|}
\hline variables & ranges of variables \\
\hline$s l$ (unit: $\mathrm{mm})$ & {$[2,25]$} \\
\hline$s w$ (unit: $\mathrm{mm})$ & {$[2,6]$} \\
\hline$x_{1}$ (unit: $\left.\mathrm{mm}\right)$ & {$[-11.5,0]$} \\
\hline$y_{1}$ (unit: $\left.\mathrm{mm}\right)$ & {$[-11.5,0]$} \\
\hline$x_{2}$ (unit: $\left.\mathrm{mm}\right)$ & {$[0,11.5]$} \\
\hline$y_{2}$ (unit: $\left.\mathrm{mm}\right)$ & {$[0,11.5]$} \\
\hline$x_{3}$ (unit: $\left.\mathrm{mm}\right)$ & {$[-11.5,0]$} \\
\hline$x_{4}$ (unit: $\left.\mathrm{mm}\right)$ & {$[0,11.5]$} \\
\hline
\end{tabular}

and $\varphi$ are given in the design requirements of the antenna. Gvariance $_{(\theta, \varphi)}$, ARvariance $_{(\theta, \varphi)}$ and $V S W$ Rvariance are the variances of gain, axial ratio and VSWR respectively over the frequency band.

In order to ensure the robustness of antenna, according to mathematical theory, we take the variance of each antenna performance in every direction and over the frequency band as objective. The smaller the variances are, the more robust the antenna is.

Therefore, details of the objective are shown as follows:

$$
\begin{aligned}
& \operatorname{Gvariance}_{(\theta, \varphi)} \\
& =\frac{1}{\operatorname{len}(f r)} \sum_{f r}\left(\operatorname{Gain}_{(\theta, \varphi, f r)}-\operatorname{Mean}_{(\theta, \varphi)}\right)^{2} \\
& \operatorname{Mean} G_{(\theta, \varphi)} \\
& =\sum_{f r} \frac{\operatorname{Gain}_{(\theta, \varphi, f r)}}{\operatorname{len}(f r)} \\
& \operatorname{ARvariance}_{(\theta, \varphi))} \\
& =\frac{1}{\operatorname{len}(f r)} \sum_{f r}\left(\operatorname{Axial}_{(\theta, \varphi, f r)}-\operatorname{Mean} A R_{(\theta, \varphi)}\right)^{2} \\
& \operatorname{Mean} A R_{(\theta, \varphi)} \\
& =\sum_{f r} \frac{\operatorname{Axial}_{(\theta, \varphi, f r)}}{\operatorname{len}(f r)} \\
& \text { VSWRvariance } \\
& =\frac{1}{\operatorname{len}(f r)} \sum_{f r}\left(V S W R_{f r}-M e a n V S W R_{f r}\right)^{2} \\
& M e a n V S W R_{f r}=\sum_{f r} \frac{V S W R_{f r}}{\operatorname{len}(f r)}
\end{aligned}
$$

where $f r$ stands for a single frequency point, and $l e n_{(f r)}$ is the number of points over frequency band. Therefore, $\operatorname{Gain}_{(\theta, \varphi, f r)}, \operatorname{Axial}_{(\theta, \varphi, f r)}$ and $V S W R_{(f r)}$ represent the gain, axial ratio and VSWR respectively in direction $(\theta, \varphi)$ and at frequency point $f r$.

The microstrip antenna design problem in this paper only involve gain and VSWR. Thus, the detail of objective is shown in Eq. $(\underset{f}{f}(\dot{\vec{x}})=V S W$ Rvariance

$$
+\sum_{\theta=-180^{\circ}}^{\theta=180^{\circ}}\left(\text { Gvariance }_{\left(\theta, 90^{\circ}\right)}\right)
$$

where

$\vec{x}$ is the solution vector;

$\varphi=90^{\circ}$;

$\theta=-180^{\circ},-175^{\circ}, \ldots, 175^{\circ}, 180^{\circ}$;

$f r=2350 \mathrm{MHz}, 2400 \mathrm{MHz}, 2450 \mathrm{MHz}$;

Details of Fvariance $_{\left(\theta, 90^{\circ}\right)}$ and VSWRariance are given in Eq. (7).

2) Constraints: According to Table I, we also set constraints on the gain and VSWR of the antenna design problem as shown in Eq. (9):

$$
\begin{aligned}
& \operatorname{gGain}_{\left(\theta, 90^{\circ}, f r\right)}(\vec{x})=-\operatorname{Gain}_{\left(\theta, 90^{\circ}, f r\right)} \leq 0 \\
& g V S W R_{f r}(\vec{x})=V S W R_{f r}-2 \leq 0
\end{aligned}
$$

3) COP of Antenna Problem: From the above, the antenna design problem is converted to a COP as follows:

$$
\begin{array}{ll}
\text { min } & f(\vec{x})=V S W \text { Rvariance } \\
& +\sum_{\theta=180^{\circ}}^{\theta=-180^{\circ}}\left(\text { Gvariance }_{\left(\theta, 90^{\circ}\right)}\right) \\
\text { st }: & g_{\text {Gain }_{\left(\theta, 90^{\circ}, f r\right)}(\vec{x})=- \text { Gain }_{\left(\theta, 90^{\circ}, f r\right)} \leq 0} \\
& g \operatorname{VWW}_{f r}(\vec{x})=\operatorname{VSW}_{f r}-2 \leq 0 \\
& \mathbf{X}=\{\vec{x} \mid \vec{l} \leq \vec{x} \leq \vec{u}\} \\
& \vec{x}=\left(s l, s w, x_{1}, y_{1}, x_{2}, y_{2}, x_{3}, x_{4}\right) \\
& \vec{l}=(2,2,-11.5,-11.5,0,0,-11.5,0) \\
& \vec{u}=(25,6,0,0,11.5,11.5,0,11.5)
\end{array}
$$

\section{VERIFYING THE ROBUST TECHNIQUE}

\section{A. Setting DE Parameters}

Differential evolution with the strategy $\mathrm{DE} / \mathrm{rand} / 1 / \mathrm{bin}$ is employed to solve the problem. The algorithm parameter settings are listed as follows:.

(1) Evolutionary generations $\mathrm{T}=500$.

(2)Population size NP $=50$.

(3) Crossover rate $\mathrm{CR}=0.9$.

(4) Scaling factor $F=0.5$

\section{B. Results and Discussion}

The electromagnetic simulation software Ansoft HFSS is adopted for evaluating the antenna performance during the run of the DE.

Here, we apply two other objective techniques, which are usually adopted to generate feasible solutions for antenna problems, to compare with our objective technique. These two objective techniques are shown as follows:

(1) The objective is defined as the sum of gain results over the directional region and the frequency band [27]. Therefore, when the solution satisfies constraints, this objective continues to find a solution with bigger gain results, see Eq. (11):

$$
f(\vec{x})=\sum_{\theta=-180^{\circ}}^{\theta=180^{\circ}} \sum_{2350 M H z}^{2450 M H z}-\operatorname{Gain}_{\left(\theta, 90^{\circ}, f r\right)}
$$

(2)The objective is zero. When the solution fits constraints, the evolutionary process stops, see Eq. (12):

$$
f(\vec{x})=0
$$

In short, we denote our objective technique variance_tec in Eq. (6) and other two objective techniques max_gain_tec in Eq. (11), feasible_only_tec in Eq. (12) respectively.

Differential evolution runs for the three objective techniques variance_tec, max_gain_tec and feasible_only_tec. The geometric structures of the three evolved antenna solutions are shown in Fig. 3, Fig. 4 and Fig. 5, respectively. And the variable values are $(17.2,2.5,-11.2,-1.5,6.1,9.1,-6,7.6)$, $(16.1,2.3,-7.8,-3,8.6,7.4,-8.3,9.5)$ and $(16.9,2,-7.9,-2.9,7.8,1.3$,$8.4,9.2)$, respectively. 


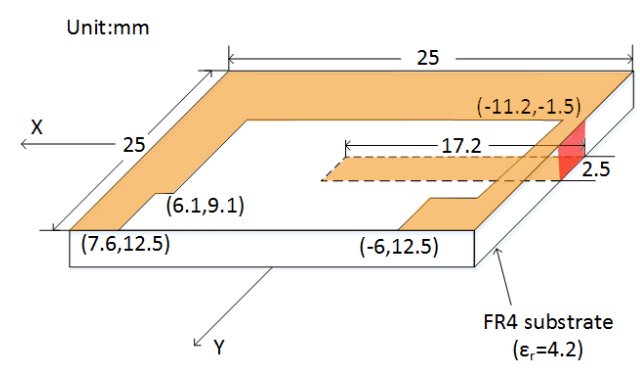

Fig. 3. Geometric structure of the evolved antenna with variance_tec

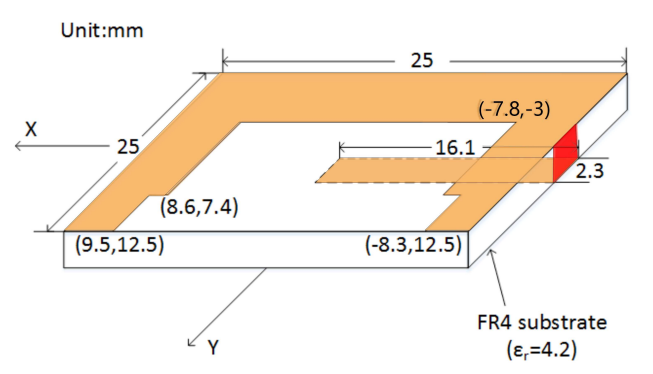

Fig. 4. Geometric structure of the evolved antenna with max_gain_tec

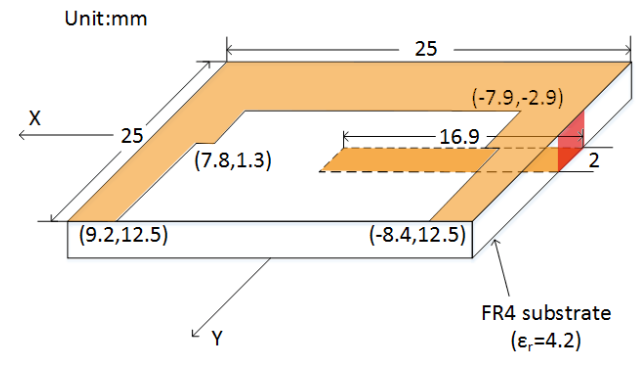

Fig. 5. Geometric structure of the evolved antenna with feasible_only_tec

1) Performances of evolved antennas: VSWRs of the three evolved antennas by the three objective technologies variance_tec, max_gain_tec and feasible_only_tec are all less than 2 in Fig. 6, and gains are greater than 0 in Fig. 7. All of the three evolved antennas satisfy the requirements. Fig. 8 shows the radiation patterns with the co-polarization and cross-polarization components of the three evolved antennas. Note the differences among the three patterns are very small since all antennas in the solution space in Eq. 5 are planar dipole antennas and with a fixed size $25 \mathrm{~mm} * 25 * 1.6 \mathrm{~mm}$, in this way, they have roughly fixed patterns.

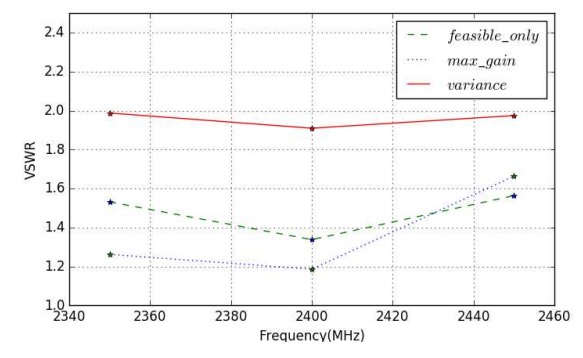

Fig. 6. VSWRs of evolved antennas at $2350 \mathrm{MHz}, 2400 \mathrm{MHz}$ and $2450 \mathrm{MHz}$

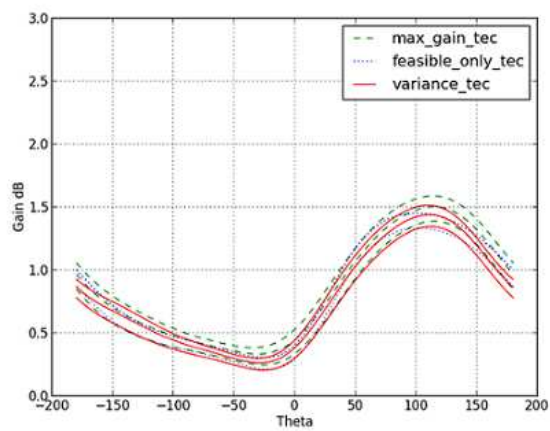

Fig. 7. Gains of evolved antennas at $2350 \mathrm{MHz}, 2400 \mathrm{MHz}$ and $2450 \mathrm{MHz}$ $\left(-180^{\circ} \leq \theta \leq 180^{\circ}\right)$
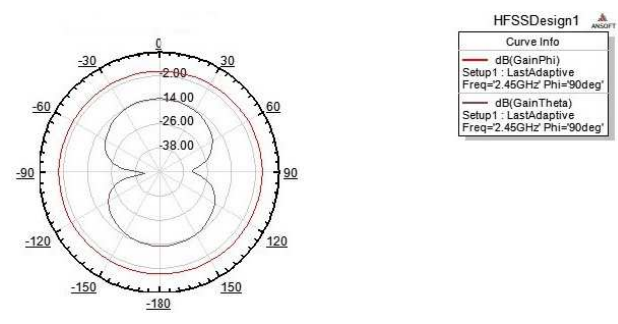

variance_tec
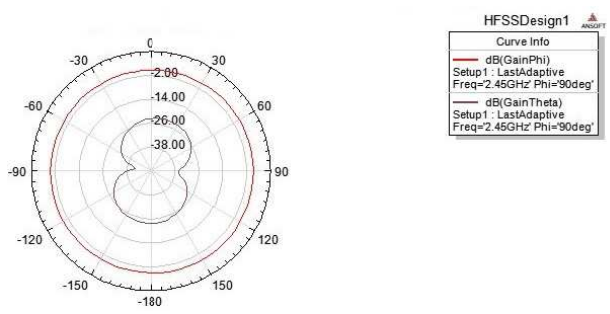

max_gain_tec
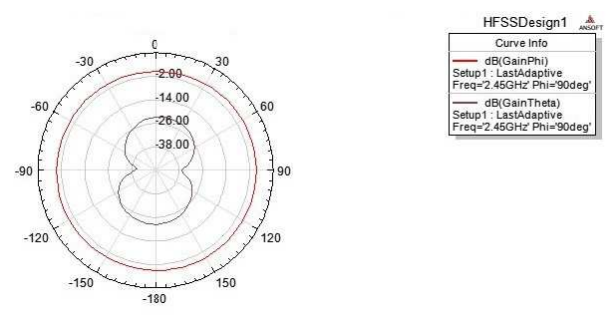

feasible_only_tec

Fig. 8. Radiation patterns of evolved antennas with the co-polarization and cross-polarization components.

2) Comparison of VSWR bandwidth: We assume that the antenna can work in a real environment when its VSWR is less than 3. Based on this assumption, obtaining bandwidth from variance_tec, max_gain_tec and feasible_only_tec are $430 \mathrm{MHz}, 320 \mathrm{MHz}$ and $380 \mathrm{MHz}$ respectively as shown in Fig. 9. It indicates that the antenna evolved by the proposed technique variance_tec has broader bandwidth than the two others. Note that VSWRs shown in Fig. 9 have slight differences with those in Fig. 6 at same frequency points. The differences are caused by the numerical computation of electromagnetic field in Ansoft HFSS, and it is trivial. 


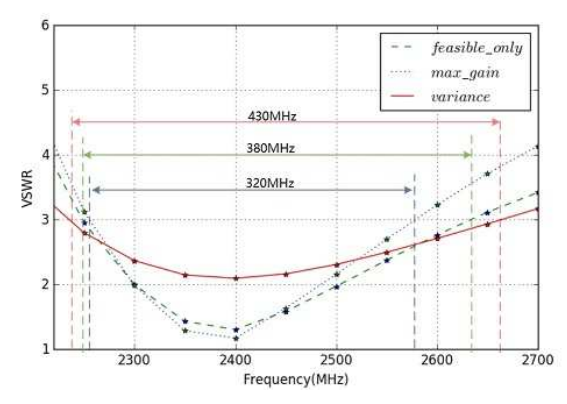

Fig. 9. Comparison of VSWR bandwidth among variance_tec,max_gain_tec and feasible_only_tec from $2200 \mathrm{MHz}$ to $2700 \mathrm{MHz}$

3) Comparison the robustness of three solutions: To verify the effectiveness of the proposed objective technoque in enhancing the robustness, we compare the robustness of the three antennas evolved by the three objective technologies variance_tec, max_gain_tec and feasible_only_tec respectively. The tolerance error of fabricating a patch antenna is usually about $\pm 0.1 \mathrm{~mm}$. In the paper, we suppose that the tolerance error is $\pm 1 \mathrm{~mm}$, that is, the range of perturbation of each design variable is $2 \mathrm{~mm}$. Four perturbations $0.5 \mathrm{~mm}, 1 \mathrm{~mm}$, $1.5 \mathrm{~mm}$ and $2 \mathrm{~mm}$ are adopted to simulate the range of $2 \mathrm{~mm}$ perturbation in the engineering fabrication. We apply the orthogonal experiment design method [19] to simulate the perturbation distribution for these four perturbations. The solution vector of antenna design problem has eight design variables and we use two levels in this paper, sixteen perturbed antennas are generated in each orthogonal experiment according to $L_{16}\left(2^{8}\right)$ orthogonal array. Altogether we generated sixty four perturbed antennas in the perturbation distribution simulation. The perturbation distribution simulation is performed on the three evolved antennas respectively. The average deviation of each performance (VSWR, gain, and so on) of the perturbed antennas from each of the evolved antenna is calculated to show the robustness of the evolved antenna. Formulations of the average deviations are shown as follows:

$$
\begin{aligned}
& V S W R \_a v g \_d e v i a t i o n_{f r} \\
& =\frac{\sum_{r=0.5,1,1.5,2} \sum_{i=1}^{16} \mid V S W R_{(r, i, f r)}-V S W R_{-} \text {evolved }_{f r} \mid}{64} \\
& \text { Gain_avg_deviation }(\theta, f r)^{=\frac{\sum_{r=0.5,1,1.5,2} \sum_{i=1}^{16} \mid \text { Gain }_{\left(\theta, 90^{\circ}, r, i, f r\right)}-\text { Gain_evolved }_{\left(\theta, 90^{\circ}, f r\right)} \mid}{64}}
\end{aligned}
$$

where

$V S W R_{(r, i, f r)}$ is the VSWR of the perturbed antenna with perturbation range $r$ at $i$ th perturbation at frequency $f r$.

$V S W R \_$evolved $f_{r}$ is the VSWR of evolved solution at frequency $f r$.

$\operatorname{Gain}_{\left(\theta, 90^{\circ}, r, i, f r\right)}$ is the gain of the perturbed antenna with perturbation range $r$ at $i$ th perturbation at frequency $f r$ in direction $\left(\theta, 90^{\circ}\right)$.

Gain_evolved $\left(\theta, 90^{\circ}, f r\right)$ is the gain of evolved solution at frequency $f r$ in direction $\left(\theta, 90^{\circ}\right)$.

$\theta=-180^{\circ},-175^{\circ}, \ldots, 175^{\circ}, 180^{\circ}$.

Note The smaller the avg_deviation are, the more robust the solution is.

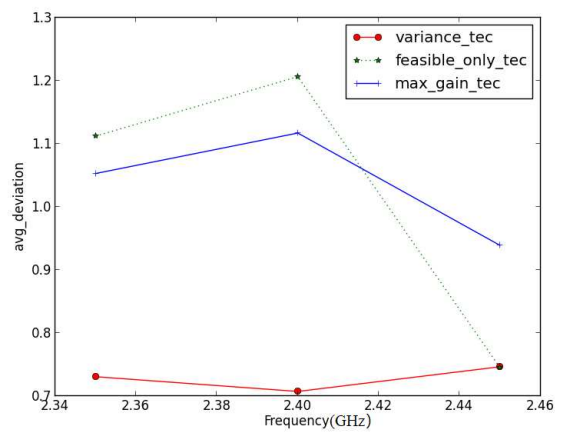

Fig. 10. Comparison of VSWR avg_deviation

TABLE III

QUANTITATIVE RESULT OF VSWR

\begin{tabular}{l|c|c|c|}
\hline & $2.35 \mathrm{GHz}$ & $2.40 \mathrm{GHz}$ & $2.45 \mathrm{GHz}$ \\
\hline variance_tec & 0.7301 & 0.7067 & 0.7458 \\
\hline max_gain_tec & 1.0523 & 1.1165 & 0.9384 \\
\hline feasible_only_tec & 1.1116 & 1.2060 & 0.7445 \\
\hline
\end{tabular}

VSWR_avg_deviations of the perturbed antennas from the three evolved antennas are calculated respectively. They are listed in Table III and plotted in Fig. 10. The VSWR_avg_deviations from the proposed technique(variance_tec), are smaller than the other two objective techniques(max_gain_tec and feasible_only_tec). It demonstrates that the proposed objective technique can generate a more robust solution than the other two techniques in VSWR.

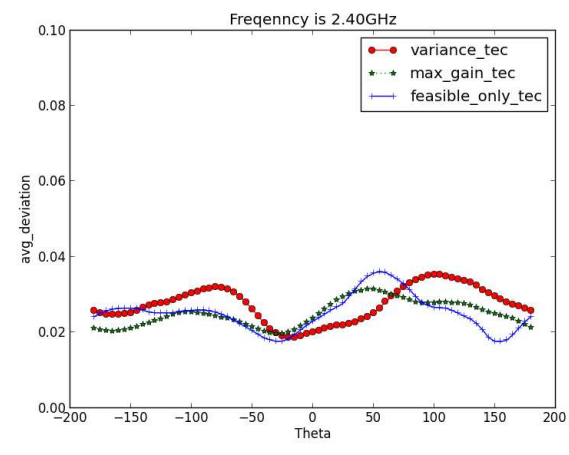

Fig. 11. Gain avg_deviation of perturbed antennas among three objectives at $2.40 \mathrm{GHz}$

TABLE IV

THE AVERAge DEVIATION VALUE OF GAIN OVER THE PERTURbation RANGE

\begin{tabular}{c|c|c|c|}
\hline & variance_tec & max_gain_tec & feasible_only_tec \\
\hline \multirow{2}{*}{$2.40 G H z$} & 0.0273 & 0.0249 & 0.0251 \\
& $-15.6(d B)$ & $-16.0(d B)$ & $-16.0(d B)$ \\
\hline
\end{tabular}

Notably, all antennas in the solution space in Eq. 5, which are planar dipole antennas with a fixed size $25 \mathrm{~mm} * 25 * 1.6 \mathrm{~mm}$, have a roughly fixed pattern. The evolved antennas by the three objective techniques and the perturbed antennas around the evolved antennas both belong to the solution space. They all 
have the roughly fixed pattern. In this way, it is easy to deduce that the three evolved antennas have very small differences of gain deviations, which means that the gains of the evolved antennas are all robust in this sense. To verify this deduction, the average deviations of gains of the perturbed antennas from the three evolved antennas are also calculated respectively at 2.40GHz. Fig. 11 show the Gain_avg_deviations over the range $\theta \in\left[-180^{\circ}, 180^{\circ}\right]$ at $2.40 \mathrm{GHz}$. The average values of Gain_avg_deviation over $\theta \in\left[-180^{\circ}, 180^{\circ}\right]$ are listed in Table IV. It can be observed that there are very small differences among three techniques in gain deviations, which are all less than $-15 d B$. That is, the gains of the evolved antennas by the three objective techniques are all robust at $2.40 \mathrm{GHz}$.

Suppose that the size of the antennas in the solution space is changeable, not with the fixed size $25 \mathrm{~mm} * 25^{*} 1.6 \mathrm{~mm}$, and the shape of the antennas is arbitrary, not the planar dipole antennas. Then the radiation patterns of the antennas are no longer fixed. The gain robustness of the evolved antennas are no longer definite. Therefore, the robustness-enhancing technique should still be applicable to gain on the above supposition, but which is not verified due to the time-expensive in this paper. The verification and the challenge of the timeexpensive will be our future work.

\section{Discussion of computational cost}

In an evolutionary robust optimization, evaluation of a solution $\mathbf{x}_{0}$ is usually implemented by evaluating a number of perturbed solutions $T$ ( e.g., the perturbations $T=64$ in this paper) around the solution $\mathbf{x}_{0}$. The average ( or the worst, etc ) value of evaluations of perturbed solutions is usually considered as the evaluation value of the solution $\mathbf{x}_{0}$. Therefore, an evolutionary robust optimization consumes $T$ times computational cost of an optimization without robust consideration ( denoted as evolutionary non-robust optimization ). The proposed technique variance_tec in this paper is actually an evolutionary non-robust optimization without additional computation. However, it can evolve a more robust antenna than other evolutionary non-robust optimization techniques, such as max_gain_tec and feasible_only_tec.

In the paper, only the microstrip planar dipole antennas with a size $25 \mathrm{~mm} * 25 \mathrm{~mm} * 1.6 \mathrm{~mm}$ was used to verify the robustness-enhancing technique since evolutionary antenna optimization is time-expensive. The experiments were run on a PC with 32-bit Intel(R)Core(TM) processor 2.33GHz, QuadCore, Memory 4G, and EAs evaluated the antennas by using HFSS 14.0 simulation software. An evolutionary non-robust optimization of microstrip planar dipole antenna in this paper consumed about 625 hours(nearly a month) and occupied about $210 M B$ memory to run smoothly. For optimizing an antenna with more complex shape, months even years are needed. An evolutionary robust optimization of antenna would greatly multiply the time. In this way, the verification of the robustness-enhancing technique applicable to gain and axial ratio has to be our future work. Notably, some of our current works focused on the challenge of time-expensive optimization (e.g.[28]), which are helpful to the future work.

\section{CONCLUSION}

This paper proposes a new technique to enhance the robustness of antennas by minimizing the variances of the gain, VSWR and so on over the required frequency band. Optimization of a microstrip planar dipole antenna is presented to illustrate the technique. Two other techniques have been used to compare the proposed technique. It has been shown that

1) The proposed technique is actually an evolutionary nonrobust optimization one without the extra computational overhead. However, it can generate a more robust antenna in VSWR than other evolutionary non-robust optimization techniques.

2) At the same time, the robust antenna evolved by the proposed technique has a broader frequency bandwidth than others.

We would like to pursue several topics in the future:

1) The robustness-enhancing technique is verified to be applicable to gain and axial ratio.

2) The proposed technique is applied to design robust antennas and fabricate them.

\section{REFERENCES}

[1] Z. Liu, S. Zeng, H. Li, S. Gao, and Z. Li, "Design of wide-beam antenna using dynamic multi-objective bbo/de," International Journal of Computer Applications in Technology, vol. 46, no. 4, pp. 376-382, 2013.

[2] R. Jiao, Y. Sun, J. Sun, Y. Jiang, and S. Zeng, "Antenna design using dynamic multi-objective evolutionary algorithm," IET Microwaves, Antennas \& Propagation, vol. 12, no. 13, pp. 2065-2072, 2018.

[3] D. E. Goldberg, "Genetic algorithms in search, optimization and machine learning," pp. 2104-2116, 1989.

[4] R. Storn and K. Price, "Differential evolution c a simple and efficient heuristic for global optimization over continuous spaces," Journal of Global Optimization, vol. 11, no. 4, pp. 341-359, 1997.

[5] I. Rechenberg, "Evolutionsstrategie optimierung technischer systeme nach prinzipien der biologischen evolution," AGRIS, vol. 15, 1973.

[6] J. Kennedy and R. C. Eberhart, "Particle swarm optimization," vol. 4, no. 0, pp. 1942-1948, 1995.

[7] J. M. J. W. Jayasinghe, J. Anguera, D. N. Uduwawala, and A. Andujar, "Nonuniform overlapping method in designing microstrip patch antennas using genetic algorithm optimization," International Journal of Antennas \& Propagation, vol. 2015, no. 2015, p. 8, 2015.

[8] E. E. Altshuler, "Electrically small self-resonant wire antennas optimized using a genetic algorithm," IEEE Transactions on Antennas \& Propagation, vol. 50, no. 3, pp. 297-300, 2002.

[9] G. S. Hornby, J. D. Lohn, and D. S. Linden, "Computerautomated evolution of an x-band antenna for nasa's space technology 5 mission," Evolutionary Computation, vol. 19, no. 1, pp. 1-23, 2014. 
[10] A. A. Al-Azza, F. J. Harackiewicz, and H. R. Gorla, "Covariance matrix adaptation evolutionary strategy optimization of patch antenna for wireless communication," Progress in Electromagnetics Research Letters, vol. 54, pp. 85-91, 2015.

[11] K. Choi, D. H. Jang, S. I. Kang, J. H. Lee, T. K. Chung, and H. S. Kim, "Hybrid algorithm combing genetic algorithm with evolution strategy for antenna design," IEEE Transactions on Magnetics, vol. 52, no. 3, pp. 14, 2016.

[12] W. Ding and G. Wang, "Design parameter optimization of ultra-wideband antenna using quantum-behaved particle swarm optimization," in Progress in Electromagnetic Research Symposium, 2016, pp. 3235-3241.

[13] W. C. Liu, "Design of a multiband cpw-fed monopole antenna using a particle swarm optimization approach," IEEE Transactions on Antennas \& Propagation, vol. 53, no. 10, pp. 3273-3279, 2005.

[14] A. Khidre, F. L. Kai, F. Yang, and A. Elsherbeni, "Wideband circularly polarized e-shaped patch antenna for wireless applications [wireless corner]," IEEE Antennas \& Propagation Magazine, vol. 52, no. 5, pp. 219-229, 2011.

[15] J. Guo and X. Liu, "Design of a wide beam wire antenna by differential evolution," in International Conference on Mechatronic Sciences, Electric Engineering and Computer, 2014, pp. 2694-2698.

[16] A. Mukhopadhyay, S. Manna, S. Lahiri, U. Sharma, M. Gangopadhyaya, P. Mukherjee, and B. Gill, "Bandwidth enhancement of microstrip patch antenna using curve fitting based differential evolution and cuckoo search optimization ca comparative study," in Information Technology, Electronics and Mobile Communication Conference, 2016, pp. 1-5.

[17] D. Lu, L. Wang, E. Yang, and G. Wang, "Design of highisolation wideband dual-polarized compact mimo antennas with multiobjective optimization," IEEE Transactions on Antennas \& Propagation, vol. 66, no. 3, pp. 1522 1527, 2018.

[18] J. Branke, "Creating robust solutions by means of evolutionary algorithms." in International Conference on Parallel Problem Solving From Nature, 1998, pp. 119128.

[19] S. Zeng, R. Wang, H. Shi, G. Chen, H. D. Garis, L. Kang, and L. Ding, "Both robust computation and mutation operation in dynamic evolutionary algorithm are based on orthogonal design," in Conference on Genetic and Evolutionary Computation, 2006, pp. 1437-1438.

[20] I. Paenke, J. Branke, and Y. Jin, "Efficient search for robust solutions by means of evolutionary algorithms and fitness approximation," IEEE Transactions on Evolutionary Computation, vol. 10, no. 4, pp. 405-420, 2006.

[21] S. Salomon, G. Avigad, P. J. Fleming, and R. C. Purshouse, "Active robust optimization: enhancing robustness to uncertain environments." IEEE Transactions on Cybernetics, vol. 44, no. 11, p. 2221, 2014.

[22] H. Fu, B. Sendhoff, K. Tang, and X. Yao, "Robust optimization over time: Problem difficulties and bench- mark problems," IEEE Transactions on Evolutionary Computation, vol. 19, no. 5, pp. 731-745, 2015.

[23] S. Mirjalili and A. Lewis, "Obstacles and difficulties for robust benchmark problems: A novel penalty-based robust optimisation method," Information Sciences, vol. 328, no. C, pp. 485-509, 2016.

[24] M. Asafuddoula, H. K. Singh, and T. Ray, "Sixsigma robust design optimization using a many-objective decomposition-based evolutionary algorithm," Evolutionary Computation IEEE Transactions on, vol. 19, no. 4, pp. 490-507, 2015.

[25] Y. Huang, Y. Ding, K. Hao, and Y. Jin, "A multi-objective approach to robust optimization over time considering switching cost," Information Sciences, vol. 394C395, pp. 183-197, 2017.

[26] J. J. Liang, T. P. Runarsson, E. Mezura-Montes, M. Clerc, P. N. Suganthan, C. A. C. Coello, and K. Deb, "Problem definitions and evaluation criteria for the cec 2006 special session on constrained real-parameter optimization," International Journal of Computer Assisted Radiology \& Surgery, no. 2, 2005.

[27] S. Zeng, Y. Jiang, Z. Liu, Y. Wu, D. Guo, L. Qiao, and Z. Liu, "A new wifi microstrip antenna designed by differential evolution," International Journal of Wireless \& Mobile Computing, vol. 8, no. 1, p. 45, 2015.

[28] R. Jiao, S. Zeng, C. Li, Y. Jiang, and Y. Jin, "A complete expected improvement criterion for gaussian process assisted highly constrained expensive optimization," Information Sciences, vol. 471, pp. 80-96, 2019.

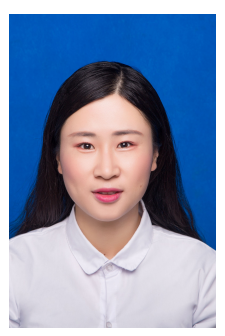

Caie Hu is currently pursuing the Ph.D degree in in control science and engineering from China University of Geoscience(Wuhan), China.

Her current research interests include data-driven surrogate-assisted evolutionary optimization with machine learning for solving problems with constraints, dynamic environments, and expensive costs, especially the antenna design problems.

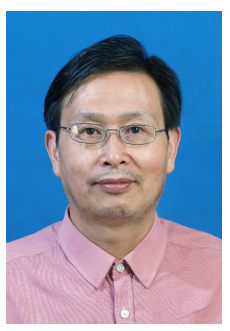

Sanyou Zeng received the M.Sc. degree in mathematics from Hunan University, Changsha, China, in 1995, and the Ph.D. degree in computer science from Wuhan University, Wuhan, China, in 2002.

$\mathrm{He}$ has been a Professor with the China University of Geosciences, Wuhan, since 2004. His current research interests include evolutionary computation with machine learning for solving problems with constraints, multi-objective, dynamic environments, and expensive costs, especially the antenna design problems. 

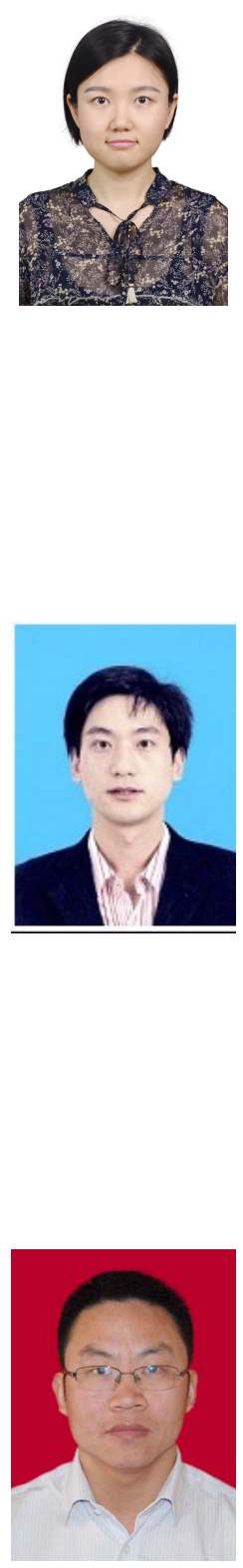

Yongzhi Sun received the Ph.D. degree from the State Key Laboratory of Millimeter Waves of Southeast university, Nanjing, China.

$\mathrm{He}$ is a Professor with Weifang University, Shandong, China. He is currently the Team Leader of the Antenna Division, Nanjin Institute of Electronic Equipment, Nanjin, China. His research interests include new concept antenna systems, RF and microwave devices, and microwave absorbing materials.

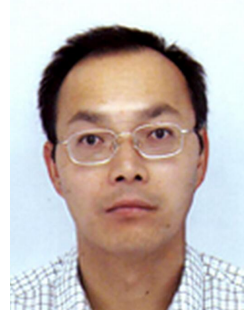

Steven Gao is a Professor and Chair of RF and Microwave Engineering at the University of Kent, UK.

His research includes antennas, smart antenna, phased array, microwave and millimeter wave circuit,satellite communication and radar. He has two books (Space Antenna Handbook, Wiley, 2012; Circularly Polarized Antennas,Wiley, 2014), over 300 papers and several patents. He is Associate Editor of IEEE Transactions on Antennas and Propagation. 